\title{
DIFERENCIAS ESTACIONALES EN LA GENERACIÓN DE ESCORRENTÍA EN UNA PEQUEÑA CUENCA DE CAMPOS ABANDONADOS EN EL PIRINEO CENTRAL
}

\author{
N. LANA-RENAULT ${ }^{(1)}$, J. LATRON $^{(2)}$, D. REGÜÉS ${ }^{(1)}$, \\ P. SERRANO ${ }^{(1)}$ y E. NADAL ${ }^{(1)}$
}

\begin{abstract}
(1) Instituto Pirenaico de Ecología (CSIC), Campus de Aula Dei, Apdo. 13034, 50080, Zaragoza.
\end{abstract}

(2) Unitat de Ciència del Sòl, Universitat de Girona, Campus de Montilivi, 17071, Girona.

Correo electrónico de contacto: noemi@ipe.csic.es

RESUMEN. Este trabajo muestra que las relaciones entre precipitación y escorrentía a escala de crecida en una pequeña cuenca de campos abandonados no son lineales y que la respuesta hidrológica se encuentra estrechamente relacionada con el estado de humedad de la cuenca. Se constata que el volumen de la precipitación y el caudal de base influyen en la magnitud del coeficiente de escorrentía mientras que la intensidad de la precipitación afecta al incremento máximo de caudal. La dinámica del nivel freático presenta una elevada estacionalidad, con un periodo seco, un periodo húmedo y un periodo de transición de condiciones secas a húmedas caracterizado por una importante variabilidad espacio-temporal de las reservas de agua de la cuenca. El análisis de la información hidrológica a escala de crecida permite diferenciar tres eventos "tipo", asociados al estado de humedad de la cuenca y que sugieren la ocurrencia de diferentes procesos dominantes de generación de escorrentía. En condiciones secas, la respuesta es limitada y la escorrentía se genera fundamentalmente por superación de la capacidad de infiltración sobre zonas degradadas de la cuenca de reducida extensión y próximas al cauce. Conforme la cuenca se humedece, dominan los procesos de escorrentía por saturación del suelo. En condiciones de máxima humedad es probable que se produzca, además, escorrentía subsuperficial.

ABSTRACT. This study shows that the relationships between rainfall and runoff at the event scale in a small farmland abandoned catchment are not linear and that the hydrological response is linked to the antecedent wetness conditions. Rainfall and baseflow discharge have a pronounce influence on the value of the runoff coef- 
ficient, while rainfall intensity affects the maximum discharge increment. Watertable data show high seasonal dynamics, with a dry period during summer, a wet period during winter and spring, and a transition period characterised by a high spatial variability. The analysis of the hydrological information at the event scale allows the distinction of three types of event, and reveals the occurrence of different runoff generation processes within the catchment. During dry conditions, the hydrological response is very low and infiltration excess over localised areas is likely to be the main runoff process. As the catchment gets wetter, saturated excess runoff (and probably subsurface flow) becomes the dominant runoff process.

Palabras clave: relaciones precipitación-escorrentía; nivel freático; estacionalidad; cuenca experimental; Pirineo Central

Key words: rainfall-runoff relationships; water-table; seasonality; research catchment; Central Pyrenees

\section{Introducción}

Los ambientes mediterráneos se caracterizan por presentar una elevada irregularidad en el régimen de las precipitaciones y una importante estacionalidad de las demandas evapotranspirativas, que explican que durante el verano se produzca un importante déficit de agua. Por ello, en estos ambientes, las relaciones entre precipitación y escorrentía son más complejas que en ambientes húmedos y, por lo tanto, la respuesta hidrológica es más difícil de predecir (Gallart et al., 1997; Piñol et al., 1997). Si bien numerosos trabajos han mostrado la falta de correspondencia que existe entre la precipitación y la escorrentía y su estrecha relación con el estado de humedad de la cuenca (Llorens y Gallart, 1992; Ceballos y Schnabel, 1998; García-Ruiz et al., 2005), pocos han estudiado la dinámica del nivel freático y su influencia sobre la respuesta hidrológica a escala de crecida.

El presente trabajo es una continuación de los trabajos realizados por García-Ruiz et al. (2005) y Seeger et al. (2004) en una pequeña cuenca de campos abandonados en el Pirineo Central, en la que se muestra el importante papel que tiene el estado de humedad de la cuenca en la repuesta hidrológica de la misma. Para la realización de este trabajo se ha contado con información relativa a las fluctuaciones del nivel freático en diferentes puntos de la cuenca. El objetivo ha sido estudiar el comportamiento hidrológico de la cuenca a lo largo del año y su relación con la evolución estacional de las reservas de agua. En primer lugar, se han examinado las relaciones entre la escorrentía y otras variables hidrológicas a escala de crecida. A continuación se ha estudiado la variabilidad espacio-temporal de la dinámica del nivel freático distinguiendo tres periodos bien diferenciados. El análisis de tres crecidas "tipo" ocurridas en diferentes condiciones de humedad ayuda a interpretar los procesos de generación de escorrentía dominantes que se producen, evidenciando la complejidad del funcionamiento hidrológico en este tipo de ambiente. 


\section{2. Área de estudio}

La cuenca experimental de Arnás $\left(2,8 \mathrm{~km}^{2}\right)$ es una cuenca de cabecera que se localiza el Sector del Flysch Eoceno Surpirenaico, entre 900 y 1400 m de altitud. La orientación NW-SE del cauce principal explica el fuerte contraste que existe entra la ladera solana, de mayor pendiente $\left(0,5 \mathrm{~mm}^{-1}\right)$, que se corresponde con un frente de cuesta, y la umbría, de pendiente más suave $\left(0,28 \mathrm{~mm}^{-1}\right)$. En la ladera solana pueden apreciarse deslizamientos superficiales todavía activos en la actualidad mientras que en la umbría antiguas lenguas de movimientos en masa profundos dan un aspecto corrugado a la ladera. Los suelos de la ladera solana son ricos en carbonatos y muestran signos de haber sufrido una intensa degradación. En esta vertiente aparecen numerosas zonas con predominio de arroyamiento difuso y/o zonas que han sido sometidas a un pastoreo más o menos intensivo. Los suelos de la vertiente umbría, en cambio, son más profundos y ricos en materia orgánica. La cuenca fue cultivada en su casi totalidad por campos en pendiente hasta mediados del siglo $\mathrm{XX}$, cuando el abandono progresivo de las actividades agrarias conllevó un proceso de recolonización vegetal natural (Genista scorpius, Buxus sempervirens, Rosa gr. canina). Actualmente en la cuenca predomina el matorral presentando, en la ladera solana, una cobertura poca densa y, en la umbría, estadios más desarrollados, con matorral denso y grandes manchas de $P$. sylvestris. En la parte inferior de las laderas, la vegetación todavía herbácea indica un abandono más reciente de los campos de cultivo.

El clima es de tipo submediterráneo con influencias atlánticas, especialmente en invierno. La precipitación media anual en el periodo 1999-2006 fue $926 \mathrm{~mm}$, con dos estaciones más lluviosas, una en primavera y otra en otoño, y una estación seca en verano, si bien en esta época del año las tormentas de tipo convectivo pueden ser relativamente frecuentes. La escorrentía anual media durante el mismo periodo fue $238 \mathrm{~mm}$, es decir, un $26 \%$ de la lluvia registrada.

\section{Metodología y equipamiento}

La cuenca está equipada (Fig. 1) con tres pluviómetros de balancín que registran en continuo la precipitación caída en forma de lluvia, una estación meteorológica completa y una estación de aforo con dos sensores (uno de presión y otro de ultrasonidos) que miden en continuo la altura de la lámina de agua. En la estación de aforo también hay instalados un turbidímetro y un conductímetro para medir en continuo, respectivamente, la exportación del sedimento en suspensión y en disolución. Para estudiar la dinámica del nivel freático, se dispone de una red de siete piezómetros, localizados en la cuenca siguiendo un criterio topográfico, en zonas en las que se ha observado en algún momento del año procesos de encharcamiento. En cada piezómetro, cuya profundidad varía entre 0,75 y 2,25 m, hay instalado un sensor de presión que almacena las fluctuaciones del nivel freático cada 20 minutos. Para el presente estudio, se han utilizado datos de precipitación y caudal de 75 crecidas ocurridas entre los años hidrológicos 1999 y 2006. El análisis de la dinámica del nivel freático ha contado con información de tres años hidrológicos completos, de 2004 a 2006. 


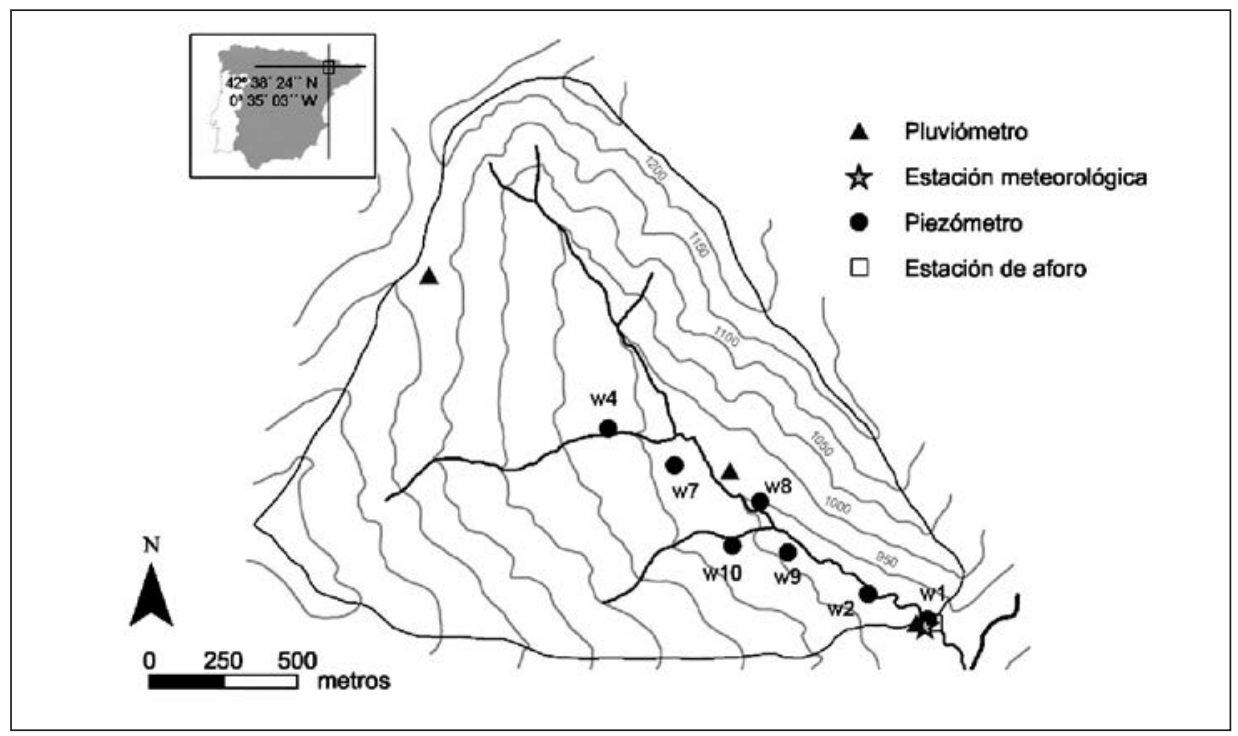

Figura 1. Dispositivo experimental de la cuenca de Arnás

\section{Resultados}

La Fig. 2, en la que se presenta la relación, a escala de crecida, entre la precipitación $(\mathrm{P})$ y la escorrentía (E), distinguiendo entre el otoño, el invierno, la primavera y el verano, sugiere que existen diferencias estacionales en la respuesta hidrológica de la cuenca. En otoño es cuando se ha registrado un mayor número de crecidas, si bien la escorrentía generada ha sido muy variable $\left(\mathrm{r}^{2}=0,34 ; p<0,01\right)$. Así, eventos en torno a 35 $\mathrm{mm}$ han podido generar indistintamente una respuesta muy limitada $(\mathrm{E}<1 \mathrm{~mm})$ o una respuesta elevada $(\mathrm{E}>7 \mathrm{~mm})$. La relación P-E mejora cuando se consideran los eventos invernales $\left(\mathrm{r}^{2}=0,61 ; p<0,01\right)$. Al contrario de lo que ocurre en otoño, en invierno, precipitaciones moderadas $(\mathrm{P}>10 \mathrm{~mm})$ siempre han generado una escorrentía significativa (E>1 mm). Sin embargo, en esta época del año, los eventos pluviométricos suelen ser de menor magnitud y, por lo tanto, las escorrentías generadas más moderadas. En primavera, la relación P-E es particularmente buena $\left(\mathrm{r}^{2}=0,76 ; p<0,01\right)$. La mayor pendiente de la recta de regresión indica que, en esta época del año, la cuenca es cuando más reacciona ante las precipitaciones. Por último, se observa que la respuesta hidrológica en verano es muy limitada y completamente independiente de las precipitaciones (la relación P-E no es significativa).

En la Fig. 3 se ha representado la evolución estacional del coeficiente de escorrentía (CE), definido como el ratio entre $\mathrm{P}$ y E, de las 75 crecidas analizadas. También se han incluido eventos pluviométricos de moderada magnitud $(\mathrm{P}>10 \mathrm{~mm})$ que no generaron respuesta hidrológica. La figura muestra, en primer lugar, la extremada variabilidad que presenta el CE a lo largo del año hidrológico. Por otra parte, se observa que el núme- 

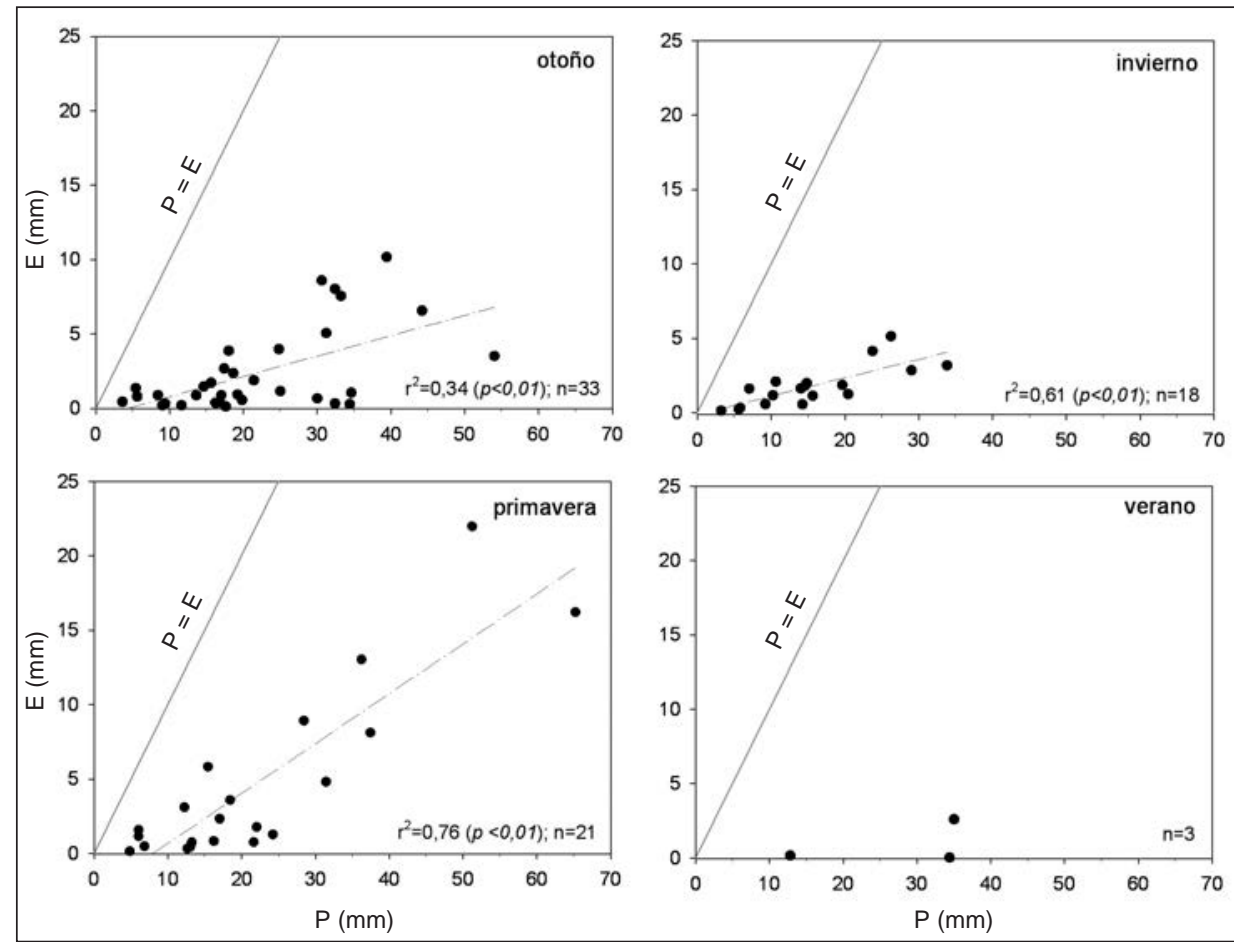

Figura 2. Relación entre precipitación $(P)$ y escorrentía $(E)$ distinguiendo entre el otoño, el invierno, la primavera y el verano (periodo 1999/2000-2006/07)

ro de eventos sin respuesta es muy importante en verano y, cuando la hay, ésta es limitada. En cambio, en invierno y en primavera, la respuesta suele ser mayor, con CE superiores a 0,03 , pudiendo alcanzar en primavera valores próximos a 0,4. En otoño, la respuesta es muy variable ya que hay eventos que no generan escorrentía o una escorrentía mínima, especialmente al principio de la estación, y eventos con CE elevados, incluso superiores a los del invierno. Un patrón similar ha sido observado en otras cuencas del ámbito mediterráneo (Gaillard et al., 1995; Latron et al., 2008). Por ejemplo, Gaillard et al. (1995) muestran que la respuesta hidrológica en la cuenca de Vaubarnier (SE de Francia) es limitada a finales del verano y principios del otoño $(\mathrm{CE}<0,05)$ mientras que en invierno los coeficientes de escorrentía varían entre el 0,15 y el 0,50, pudiendo alcanzar en determinadas ocasiones valores de 0,7. Tal y como sugieren Gallart et al. (1997), la evolución estacional del CE a escala de crecida puede relacionarse con la estacionalidad de la dinámica de las reservas de agua de la cuenca. A principios del año hidrológico (octubre), las precipitaciones abundantes, ocurridas después de un periodo estival seco, no generan respuesta alguna o una respuesta muy limitada, ya que contribuyen a la recarga del contenido de agua en el suelo y del nivel freático. A medida que la cuenca se humedece, la respuesta hidrológica aumenta y representa una mayor fracción de la precipitación. 


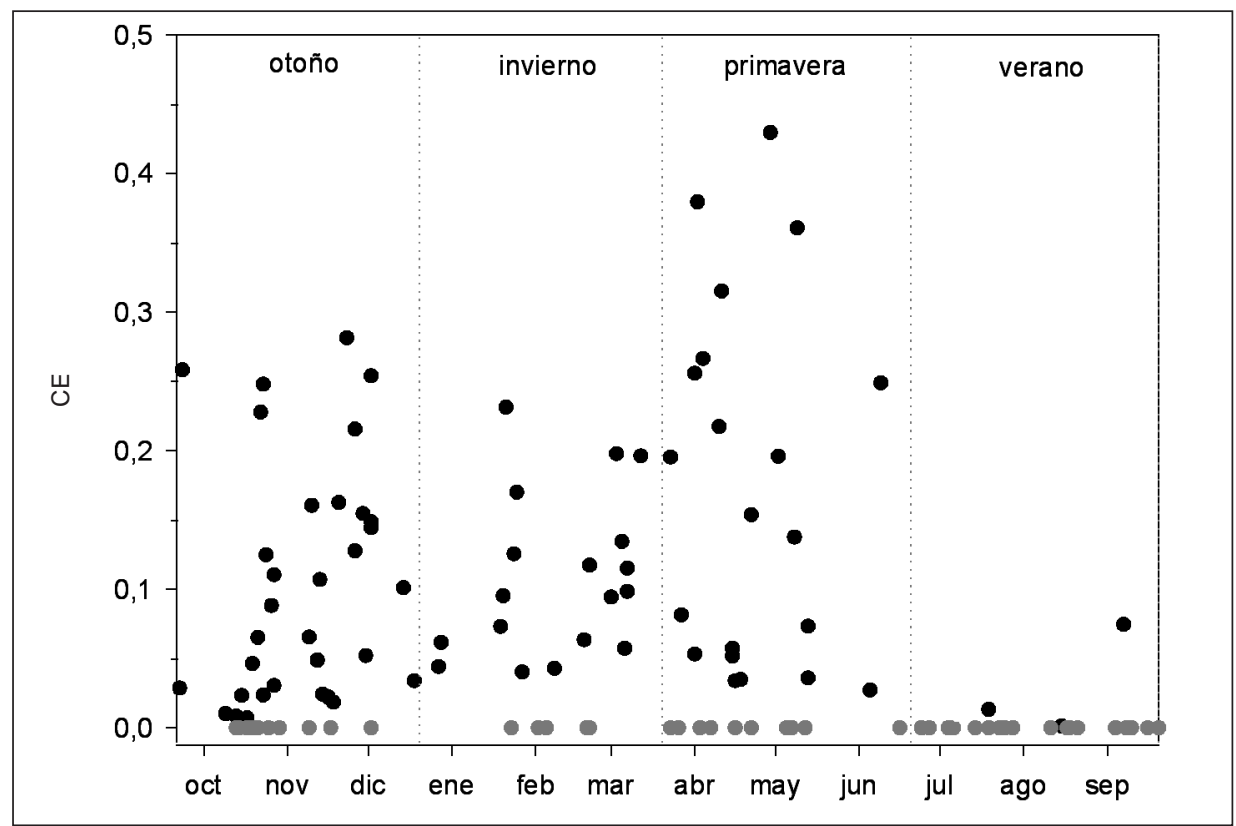

Figura 3. Evolución estacional del coeficiente de escorrentía (CE) de las 75 crecidas analizadas. En gris se han marcado eventos pluviométricos moderados (P>10 mm) que no han generado respuesta hidrológica

Con el fin de estudiar la influencia que pueden ejercer otros factores sobre la variabilidad de la respuesta hidrológica de la cuenca, se ha realizado un análisis de correlación entre el CE, la precipitación total, la intensidad máxima de la precipitación y el caudal específico de base (Tabla 1). Los resultados muestran que la correlación entre el CE y el volumen de precipitación es significativa, lo que sugiere que existe una tendencia positiva entre estas dos variables, aunque la dispersión de los puntos es muy importante $(\mathrm{R}=0,28 ; p<0,05)$. La correlación entre el $\mathrm{CE}$ y la intensidad máxima de la precipitación no resulta significativa, confirmando la escasa influencia que tiene esta variable sobre la respuesta hidrológica en cuencas cubiertas por vegetación (Hewlett et al., 1977; Serrano-Muela et al., 2008). No obstante, la ausencia de una relación significativa entre estas dos variables a escala de evento no implica que la intensidad de la precipitación no pueda ser relevante localmente en zonas desprovistas de vegetación (Gallart et al., 2005). Por último, la correlación entre el CE y el caudal de base, considerado como indicador del estado de humedad de la cuenca, es significativa y positiva $(\mathrm{R}=0,38 ; p<0,01)$, si bien el moderado valor de $\mathrm{R}$ indica que el peso de esta variable no es claro. Este resultado, similar al observado en otras cuencas mediterráneas (Cosandey, 1993; Latron et al., 2008), contrasta con el obtenido en ambientes más húmedos, en los que el $\mathrm{CE}$ aparece estrechamente relacionado con el caudal de base previo a la precipitación (Cappus, 1960; Jordan, 1994). 
Tabla 1. Coeficientes de correlación lineal entre el coeficiente de escorrentía de crecida (CE), el incremento máximo de caudal (incQ), y otras variables hidrológicas: precipitación $(P)$, intensidad máxima de precipitación (IP5) y caudal específico de base al inicio del evento $\left(Q_{0}\right)$. Las correlaciones son significativas al 95\% (*) o al 99\% (**)

\begin{tabular}{|c|c|c|c|}
\hline & $\mathbf{P}(\mathbf{m m})$ & $\mathbf{I P 5}\left(\mathbf{m m ~ h} \mathbf{h}^{\mathbf{1}}\right)$ & $\mathbf{Q}_{\mathbf{0}}\left(\mathbf{l s}^{\mathbf{- 1}} \mathbf{k m}^{\mathbf{2}}\right)$ \\
\hline $\mathrm{CE}$ & $0,28 *$ & $-0,04$ & $0,38^{* *}$ \\
incQ $\left(1 \mathrm{~s}^{-1} \mathrm{~km}^{-2}\right)$ & $0,63 * *$ & $0,48^{* *}$ & $-0,05$ \\
\hline
\end{tabular}

De la misma manera, se ha estudiado cómo estas variables afectan a la intensidad de la respuesta hidrológica, reflejada en el incremento máximo de caudal (incQ). La relación entre incQ y la precipitación presenta un coeficiente de correlación más elevado que cuando se consideraba el coeficiente de escorrentía $(\mathrm{R}=0,63 ; p<0,01)$. Por otra parte, la intensidad de la precipitación aparece como una variable significativa y con un coeficiente de correlación moderado $(\mathrm{R}=0,48 ; p<0,01)$. De ello se desprende que tanto el volumen como la intensidad de la precipitación influyen en la intensidad de la respuesta hidrológica a la salida de la cuenca. La relación entre incQ y la precipitación es acorde con los resultados obtenidos en cuencas de ambientes húmedos (Hewlett et al., 1977; Jordan, 1994) mientras que la influencia de la intensidad de la precipitación es más característica de cuencas en zonas poco permeables (Ceballos y Schnabel, 1998). Por último, la relación entre incQ y el caudal de base no resulta significativa. La ausencia de correlación entre estas dos variables, que también ha sido observada por Latron et al. (2008) en el Pirineo Oriental, sugiere que el estado de humedad de la cuenca no tiene un peso claro a la hora de explicar la intensidad de la respuesta hidrológica de la cuenca.

En la Fig. 4 se ha representado la evolución estacional del nivel freático medio de la cuenca (calculado a partir de los datos diarios de los piezómetros w7, w8 y w9) para el año hidrológico 2006/07. También se ha representado la desviación estándar asociada a cada dato pues es indicadora de la variabilidad espacial que existe entre los tres puntos de medida. En la figura pueden distinguirse claramente tres periodos:

- Entre noviembre y finales de mayo, el nivel de la capa freática estuvo próximo a la superficie del suelo en los tres puntos de medida. Las variaciones del nivel freático en esta época del año no son significativas.

- A partir de junio y hasta el final del verano la cuenca se fue secando progresivamente debido a un aumento de la evapotranspiración. Durante este periodo el valor de la desviación estándar es más elevado debido a las diferencias temporales en el drenaje de los diferentes puntos de medida. Se observa que sólo en determinados casos el valor de la desviación estándar disminuye, coincidiendo con la ocurrencia de tormentas estivales que provocaron una elevación momentánea del nivel freático en los tres puntos de medida. 


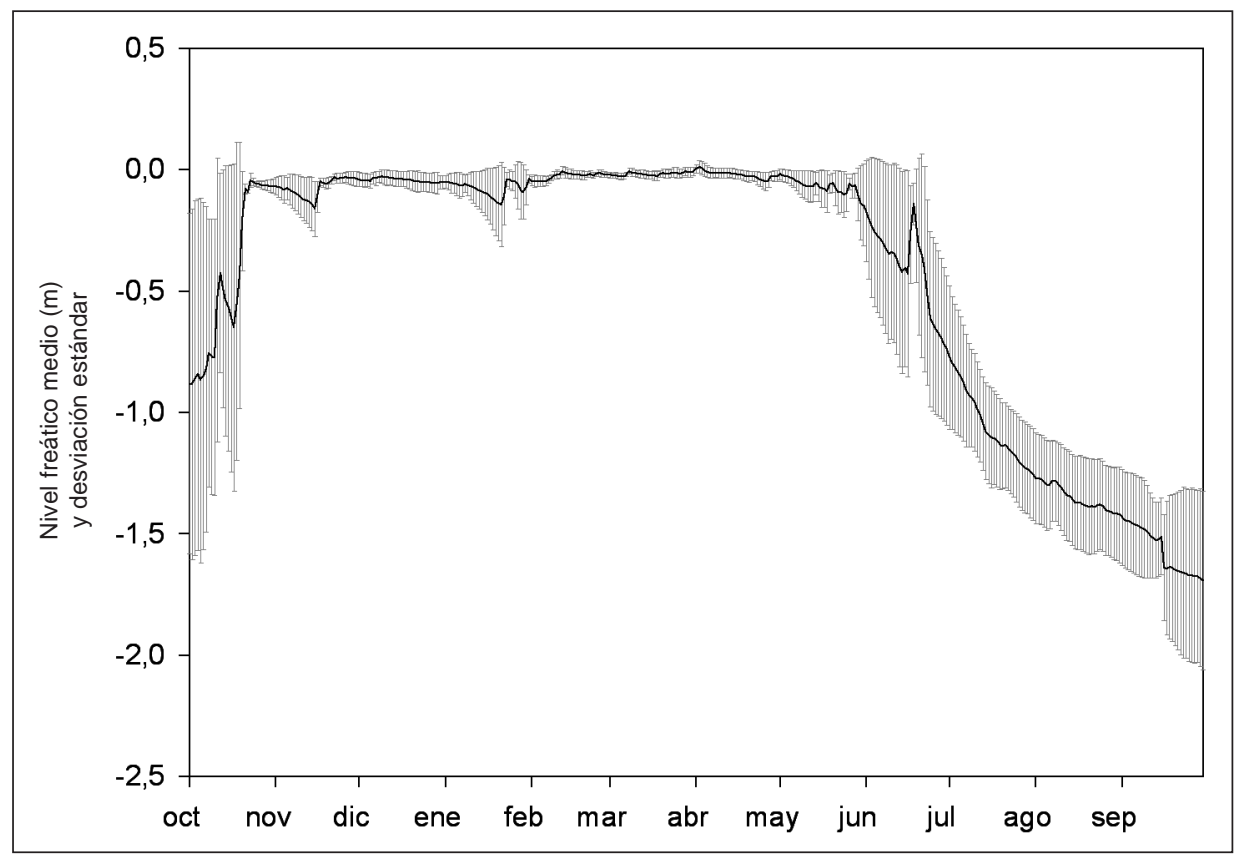

Figura 4. Evolución estacional del nivel freático medio calculado a partir de los piezómetros w7, w8 y w9 (año hidrológico 2006/07)

- Con las primeras precipitaciones de octubre y hasta que las reservas de agua quedaron restituidas se distingue un periodo de transición de condiciones secas a húmedas. En este periodo el valor de la desviación estándar es máximo, indicando una importante variabilidad espacial en el proceso de recarga de las reservas de agua de la cuenca. Por ejemplo, el nivel freático en el piezómetro w8 reaccionó rápidamente a las primeras lluvias otoñales (ascendió 1,4 m en 4 días) mientras que en el piezómetro w7 aumentó de manera más progresiva (el mismo aumento se produjo en 25 días).

Un patrón estacional similar de la dinámica del nivel freático ha sido descrito en otras cuencas experimentales. En una cuenca forestal en Georgia (EE.UU), Peters et al. (2003) observan un aumento de la capa freática tras las intensas lluvias que ocurren al final del periodo estival, y una saturación generalizada a lo largo del otoño y el invierno. En la cuenca de Cal Rodó, Latron (2003) también describe un aumento generalizado de los niveles freáticos durante el invierno y un descenso progresivo durante la primavera hasta finales del verano. Además, este autor señala que la dinámica del nivel freático presenta una importante variabilidad espacial, sobre todo durante los periodos de transición de condiciones secas a húmedas. 
En función de los diferentes estados de humedad de la cuenca (periodo seco, periodo de transición y periodo húmedo) y de las características de la precipitación, se han identificado tres tipos de respuesta hidrológica asociada a diferentes procesos de generación de escorrentía dominantes (Fig. 5). En esta figura, se ha representado, para cada crecida "tipo", el hietograma, el hidrograma y la evolución del nivel freático en los puntos w7, w8 y w9.

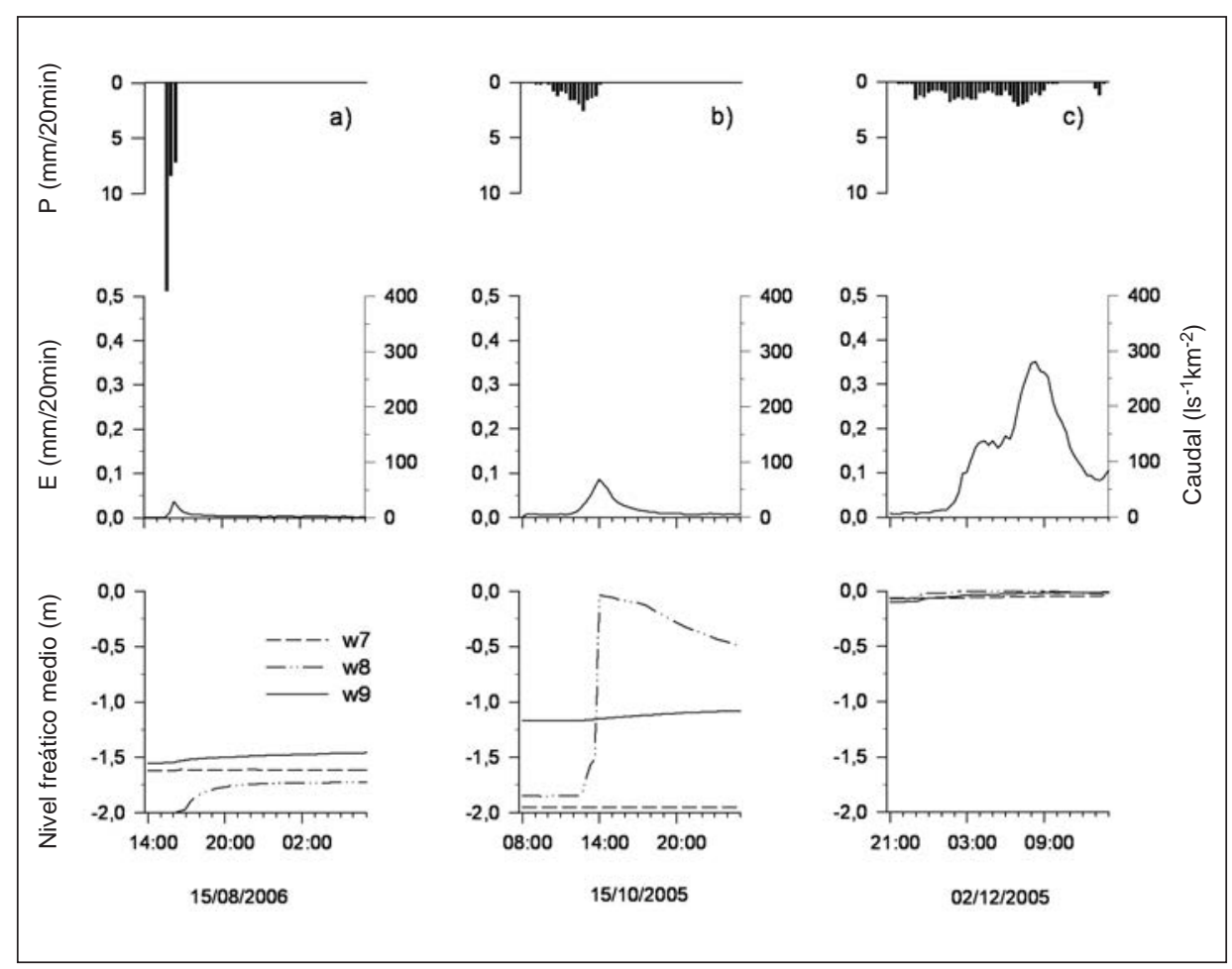

Figura 5. Precipitación $(P)$, escorrentía $(E)$ y dinámica del nivel freático en los puntos $w 7, w 8$ y w9 para tres tipos de respuesta hidrológica en la cuenca de Arnás: a) en condiciones secas; b) en condiciones de transición; c) en condiciones húmedas

\subsection{Respuesta hidrológica en condiciones secas}

El primer evento (Fig. 5a) se corresponde con una tormenta intensa que ocurrió en el verano de 2006. Al inicio del evento no había caudal de base y los piezómetros estaban secos o con los niveles freáticos muy profundos, indicando que las reservas hidrológicas de la cuenca eran mínimas. A pesar de que la precipitación fue abundante $(\mathrm{P}=34,4 \mathrm{~mm})$ e intensa (IP5>100 $\mathrm{mm} \mathrm{h}^{-1}$ ), la respuesta del caudal y del nivel freático resultó limitada. El pico de caudal no superó los $30 \mathrm{l} \mathrm{s}^{-1} \mathrm{~km}^{-2}$ y el valor del coeficiente de escorrentía fue inferior a 0,01. Asimismo, sólo se observó un ligero aumento del nivel 
freático en los puntos w8 y w9 (inferior a 0,25 m), que se produjo una hora después del inicio de la tormenta y que se prolongó varias horas más, coincidiendo con la rama descendente del hidrograma.

Este tipo de respuesta hidrológica se observa cuando la cuenca se encuentra en condiciones secas, coincidiendo generalmente con el periodo estival. En estas condiciones, las crecidas suelen ser rápidas y relativamente cortas, con tiempos de respuesta en torno a 1 hora e hidrogramas de crecida que duran menos de 3 horas. Los coeficientes de escorrentía son bajos $(C E<0,1)$ y los picos de caudal específico moderados $\left(Q_{\max }<501 \mathrm{~s}^{-1} \mathrm{~km}^{-2}\right)$. Además, los niveles freáticos apenas registran respuesta durante el evento. Todo ello sugiere que este tipo de crecidas se produzca por procesos de generación de escorrentía por superación de la capacidad de infiltración (escorrentía hortoniana) sobre zonas degradadas de la cuenca que se encuentran próximas al cauce. La reducida extensión de estas áreas explica que, en estas condiciones, los coeficientes de escorrentía sean bajos. Trabajos realizados en otras cuencas mediterráneas señalan la ocurrencia de este tipo de procesos. Así, en una cuenca de la dehesa extremeña, Ceballos y Schnabel (1998) sugieren que, en condiciones secas, se produce escorrentía hortoniana que se relacionan con hidrogramas súbitos y de corta duración. Asimismo, en la cuenca de Chicamo, en Murcia, Martínez-Mena et al. (1998) localizan este tipo de procesos sobre los suelos más degradados.

\subsection{Respuesta hidrológica en condiciones intermedias}

El evento del 15 de octubre de 2005 (Fig. 5b) ocurrió durante el periodo de transición de condiciones secas a condiciones húmedas, después de que se registraran las primeras tormentas otoñales. El caudal específico de base al inicio de la precipitación era de $51 \mathrm{~s}^{-1} \mathrm{~km}^{-2}$ y el nivel freático en w8 y w9 estaba ligeramente más elevado que en el evento descrito anteriormente, aunque los demás piezómetros se encontraban completamente secos. En estas condiciones, una precipitación de $16,2 \mathrm{~mm}$ y de baja intensidad (IP5 $<10 \mathrm{~mm} \mathrm{~h}^{-1}$ ) produjo una respuesta del caudal pequeña, con un coeficiente de escorrentía de 0,02 y un pico de caudal en torno a $701 \mathrm{~s}^{-1} \mathrm{~km}^{-2}$. La respuesta del nivel freático varió de un sitio a otro: mientras que en w9 tan sólo se elevó unos pocos centímetros, el piezómetro w8 registró una subida del nivel freático muy rápida (1,5 m en 20 min) que alcanzó la superficie del suelo en el mismo momento en el que se midió el pico de caudal, y luego fue disminuyendo progresivamente.

El hecho de que durante este tipo de crecidas el nivel freático alcance la superficie del suelo en determinados puntos de medida indica que, al menos, en algunas partes de la cuenca se forman áreas saturadas. La respuesta hidrológica en estas condiciones se genera, por lo tanto, por procesos de saturación sobre determinadas áreas de la cuenca que, junto con los procesos por superación de la capacidad de infiltración (reflejados en la ocurrencia de picos de caudal importantes cuando la precipitación es intensa), producen una respuesta del caudal superior a la observada en condiciones secas. En una cuenca mediterránea, Grésillon y Taha (1998) constatan que las crecidas pueden generarse por procesos de saturación en determinadas zonas asociadas a la aparición de capas fre- 
áticas colgadas y temporales. En la cuenca de Cal Parisa, en el Pirineo Oriental, Gallart et al. (1994) muestran la influencia de las antiguas terrazas de cultivo en la aparición de áreas saturadas. Asimismo, Martínez-Fernández et al. (2005) sugieren que, a pesar de su reducida extensión, las zonas saturadas observadas en el fondo de valle en una cuenca del Sistema Central son determinantes en la generación de escorrentía.

\subsection{Respuesta hidrológica en condiciones húmedas}

El tercer evento seleccionado (Fig. 5c) ocurrió a principios del mes de diciembre de 2005 , cuando el caudal específico de base no era muy elevado $\left(Q_{0}=51 \mathrm{~s}^{-1} \mathrm{~km}^{-2}\right)$ pero el nivel freático estaba próximo a la superficie del suelo en la mayoría de los puntos de medida. La precipitación fue abundante $(\mathrm{P}=44,8 \mathrm{~mm})$ y mostró un patrón típico invernal, con una intensidad baja (IP5<7 $\mathrm{mm} \mathrm{h}^{-1}$ ). En estas condiciones, la respuesta hidrológica fue lenta (el pico de caudal se alcanzó siete horas después del inicio de la crecida) y la rama descendente del hidrograma presentó una pendiente suave. El coeficiente de escorrentía fue moderado $(\mathrm{CE}=0,15)$ y el pico de caudal estuvo en torno a $300 \mathrm{l} \mathrm{s}^{-1} \mathrm{~km}^{-2}$.

Este tipo de crecida se observa cuando la cuenca está húmeda, es decir, cuando las reservas de agua están completamente restituidas. En estas condiciones se ha constatado que las áreas saturadas ocupan una superficie importante de la cuenca. El tiempo de respuesta, más bien lento, y la longitud de la rama descendente, que caracteriza a muchas de estas crecidas, sugiere que, además de una contribución de la escorrentía por saturación del suelo, variable en función del grado de humedad de la cuenca, puede haber una contribución de escorrentía subsuperficial. Este tipo de escorrentía se vería favorecido por la ocurrencia de precipitaciones de baja intensidad que podrían ser absorbidas por los suelos que no estuvieran saturados y que representan una gran parte de la superficie de la cuenca independientemente de su estado hidrológico. Este tipo de proceso podría también explicar los coeficientes de escorrentía especialmente elevados (CE>0,3) que se han registrado durante algunas crecidas, sobre todo al final del periodo húmedo.

Aunque los procesos de escorrentía subsuperficial han sido descritos con más frecuencia en ambientes templado-húmedos, estudios como los de Taha et al. (1997) en una cuenca mediterránea muestran cómo una proporción importante del hidrograma de crecida proviene de escorrentía subsuperficial. Asimismo, en la cuenca de Can Vila, en el Pirineo Oriental, Latron y Gallart (2007) sugieren que la escorrentía subsuperficial, junto con procesos de saturación y superación de la capacidad de infiltración, contribuyen en la respuesta hidrológica.

\section{Conclusiones}

El presente estudio muestra que, en una cuenca de montaña media afectada por una intensa actividad agraria en el pasado y sujeta a un proceso de revegetación natu- 
ral en la actualidad, las relaciones precipitación-escorrentía a escala de crecida no son lineales, y que la respuesta hidrológica presenta una notable estacionalidad, asociada al estado de humedad de la cuenca, siendo mayor en invierno y primavera, y más limitada en verano y a principios del otoño. Los análisis indican que tanto el volumen de la precipitación como el caudal de base influyen en la magnitud de la respuesta hidrológica a la salida de la cuenca, mientras que la intensidad de la precipitación afecta al incremento máximo de caudal, sugiriendo la ocurrencia de procesos de generación de escorrentía por superación de la capacidad de infiltración en determinadas zonas de la cuenca cuando ocurren lluvias intensas, especialmente en verano.

El estudio de la dinámica del nivel freático muestra que las reservas de agua de la cuenca presentan una importante estacionalidad con i) un periodo de desecamiento que se corresponde con el periodo estival, ii) un periodo de transición de condiciones secas a húmedas, caracterizado por una elevada variabilidad espacial de las reservas de agua de la cuenca y iii) un periodo húmedo durante el cual las reservas de agua están completamente restituidas.

El análisis de tres eventos "tipo" evidencia la importancia que tiene la dinámica del nivel freático en la respuesta hidrológica a la salida de la cuenca y sugiere la ocurrencia de diferentes procesos dominantes de generación de escorrentía. Así, cuando la cuenca se encuentra en condiciones secas (los piezómetros están secos o con los niveles freáticos muy profundos), las crecidas se caracterizan por una respuesta rápida y con coeficientes de escorrentía muy bajos. En estas condiciones, las crecidas se generan fundamentalmente por procesos de superación de la capacidad de infiltración en zonas degradas de la cuenca de reducida extensión y próximas al cauce. Cuando la cuenca se encuentra en un periodo de transición de condiciones secas a húmedas, durante el cual los niveles freáticos tienen valores intermedios y presentan una importante variabilidad espacial, los coeficientes de escorrentía son moderados. La aparición de algunas áreas saturadas, asociadas a un afloramiento puntual del nivel freático, sugiere la ocurrencia de procesos de generación de escorrentía por saturación del suelo y, por lo tanto, un incremento de las áreas que contribuyen a la respuesta hidrológica de la cuenca. Por último, en condiciones húmedas (cuando las reservas de agua de la cuenca están restituidas), los coeficientes de escorrentía suelen ser más elevados debido a una expansión de las áreas saturadas. Por otra parte, durante las crecidas que presentan tiempos de respuesta más largos, y que coinciden con las que tienen coeficientes de escorrentía mayores, es probable que se produzca una contribución de escorrentía subsuperficial.

Los análisis llevados a cabo en este trabajo tienen un carácter hidrométrico y constituyen una base para diagnosticar el comportamiento hidrológico de la cuenca. Este diagnóstico es necesario a la hora de aplicar modelos hidrológicos y comprobar las hipótesis asumidas en el proceso de modelización (Gallart et al., 1997; Beven y Freer, 2001). Por otra parte, de este trabajo se desprende que el funcionamiento hidrológico de este tipo de ambiente es complejo y que la cuenca de Arnás puede considerarse como un mosaico complejo con piezas muy diferentes que se activan hidrológicamente en momentos distintos, en función de la duración y la intensidad de la precipitación y el estado de humedad de la 
cuenca. Esta complejidad es el resultado de la acción antropogénica discriminada en el pasado, con áreas de uso más intensivo y otras más extensivo o conservador, dificultando sin lugar a dudas su modelización y la predicción de su respuesta hidrológica. El esquema de funcionamiento hidrológico propuesto en este trabajo se basa no obstante en hipótesis que, en ocasiones, pueden ser difíciles de verificar con los datos analizados. La obtención de información adicional (por ej., el transporte de material en disolución) permitirá diferenciar determinados procesos de generación de escorrentía (por ej., escorrentía subsuperficial). En cualquier caso, resulta necesario seguir analizando las relaciones espacio-temporales entre la precipitación, el estado de humedad de la cuenca y la escorrentía a escala de crecida, con el fin de conseguir una "conceptualización” precisa de los procesos hidrológicos dominantes en la cuenca.

\section{Agradecimientos}

Este trabajo se ha realizado con el apoyo de los siguientes proyectos de investigación: "Procesos y balances hidrológicos y de sedimentos a diferentes escalas espaciales en ambientes mediterráneos: efectos de las fluctuaciones climáticas y los cambios de uso del suelo-PROBASE" (GCL2006-11619/HID) y "Caracterización y modelización de procesos y regímenes hidrológicos en cuencas aforadas para la predicción en cuencas no aforadas-CANOA" (CGL2004-04919-C02-01/HID), ambos financiados por la CICYT. La monitorización de las cuencas ha contado con el apoyo del convenio entre el CSIC y el Ministerio de Medio Ambiente (RESEL).

\section{Referencias bibliográficas}

Beven, K. y Freer J. (2001). A dynamic TOPMODEL. Hydrological Processes, 15: 1992-2011.

CAppus, P. (1960). Bassin expérimental d'Alrance. Étude des lois de l'écoulement. Application au calcul et à la prévision des débits. La Houille Blanche A: 493-520.

Ceballos, A. y Schnabel, S. (1998). Hydrological behaviour of a small catchment in the dehesa landuse system (Extremadura, SW Spain). Journal of Hydrology, 210: 146-160.

COSANDEY, C. (1993). Forêt et écoulements: Rôle de la forêt sur la formation des crues et le bilan d'écoulement annuel. Impact d'une coupe forestière. Rapport sectoriel de recherche du Programme CEE "suivi des conséquences d'une coupe forestière sur les phénomènes hydrologiques, hydrochimiques et d'érosion solide dans un bassinversant de Lozère". 81 p. 
Gaillard, E., Lavabre, J., Isberie, C. y Normand, M. (1995). Etat hydrique d'une parcelle et écoulements dans un petit bassin versant du massif cristallin des Maures. Hydrogéologie, 4: 41-48.

Gallart, F., Llorens, P. y Latron, J. (1994). Studying the role of old agricultural terraces on runoff generation in a small Mediterranean mountainous basin. Journal of Hydrology, 159 (1-4): 291-303.

Gallart, F., LAtron, J., Llorens, P. y RABADÁ, D. (1997). Hydrological functioning of Mediterranean mountain basins in Vallcebre, Catalonia: some challenges for hydrological modelling. Hydrological Processes, 11: 1263-1272.

Gallart, F., Balasch, J.C, Regüés, D., Soler, M. y Castelltort, X. (2005). Catchment dynamics in a Mediterranean mountain environment. The Vallcebre research basins (southeastern Pyrenees) II: temporal and spatial dynamics of erosion and stream sediment transport. En: Catchment dynamics and River Processes: Mediterranean and other Climate Regions (García, C. y Batalla, R.J., Eds.). Elsevier, pp. 17-29, Amsterdam.

García-Ruiz, J.M., Arnáez, J., Beguería, S., Seeger, M., Marti, C., RegüÉs, D., LANA-RENAUlt, N. y White, S. (2005). Flood generation in an intensively disturbed, abandoned farmland catchment, Central Spanish Pyrenees. Catena, 59: 79-92.

GRESILLON, J.M. y TAHA, A. (1998). Les zones saturées contributives en climat méditerranéen: condition d'apparition et influence sur les crues. Hydrological Sciences Bulletin-des Sciences Hydrologiques, 43 (2): 267-282.

Hewlett, J.D., FoRTSON, J.C. y CunNingham, G.B. (1977). Effect of Rainfall Intensity on Storm Flow and Peak Discharge from Forest Land. Water Resources Research, 13 (2): 259-266.

JORDAN, J.P. (1994). Spatial and Temporal Variability of Stormflow Generation Processes on a Swiss Catchment. Journal of Hydrology, 153 (1-4): 357-382.

LATRON, J. (2003). Estudio del funcionamiento hidrológico de una cuenca mediterránea de montaña. Tesis Doctoral inédita. Univ. Barcelona.

LATRON, J. y GALlaRT, F. (2007). Seasonal dynamics of runoff-contributing areas in a small mediterranean research catchment (Vallcebre, Eastern Pyrenees). Journal of Hydrology, 335 (1-2): 194-206.

Latron, J., Soler, M., Llorens, P. y Gallart, F. (2008). Spatial and temporal variability of the hydrological response in a small Mediterranean research catchment (Vallcebre, Eastern Pyrenees). Hydrological Processes, 22: 775-787. 
LLORENS, P. y GALLART, F. (1992). Small basin response in a mediterranean mountainous abandoned farming area: research design and preliminary results. Catena, 19 (3-4): 309-320.

Martínez-Mena, M., Albaladejo, J. y Castillo, V. (1998). Factors influencing surface runoff generation in a Mediterranean semi-arid environment: Chicamo watershed, SE Spain. Hydrological Processes, 12: 741-754.

Martínez-Fernández, J., Ceballos Barbancho, A., Hernández Santana, V., Casado ledesma, S. y Morán Tejeda, C. (2005). Procesos hidrológicos en una cuenca forestal del Sistema Central: cuenca experimental de Rinconada. Cuadernos de Investigación Geográfica, 31: 7-25.

Peters, E., Freer, J. y Aulenbach, B.T. (2003). Hydrological Dynamics of the Panola Mountain Research Watershed, Georgia. Ground Water, 41 (7): 973-988.

Piñol, J., BeVEn, K. y FreER, J. (1997). Modelling the hydrological response of Mediterranean catchments, Prades, Catalonia. The use of distributed models as aids to hypothesis formulation. Hydrological Processes, 11 (9): 1287-1306.

Seeger, M., Errea, M.P., Beguería, S., Arnáez, J., Martí, C. y García-Ruiz, J.M. (2004). Catchment soil moisture and rainfall characteristics as determinant factors for discharge/suspended sediment hysteretic loops in a small catchment in the Spanish Pyrenees. Journal of Hydrology, 288: 299-311.

Serrano-Muela, M.P., Lana-Renault, N., NADAl-Romero, E., RegüÉS, D., LATRON, J., MARTI-BONO, C. y GARCÍA-RUIZ, J.M. (2008). Forests and water resources in mediterranean mountains: the case of the Spanish Pyrenees. Mountain Research and Development, 28(3).

TAHA, A., Gresillon, J.M. y ClothieR, B.E. (1997). Modelling the link between hillslope water movement and stream flow: application to a small Mediterranean forest watershed. Journal of Hydrology, 203: 11-20. 
